\title{
Stimulated Raman Adiabatic Passage in Optomechanics
}

\author{
Vitaly Fedoseev $\odot,{ }^{1, *}$ Fernando Luna, ${ }^{2}$ Ian Hedgepeth, ${ }^{2}$ Wolfgang Löffler $\odot,{ }^{1}$ and Dirk Bouwmeester ${ }^{1,2}$ \\ ${ }^{1}$ Huygens-Kamerlingh Onnes Laboratorium, Leiden University, 2333 CA, Leiden, Netherlands \\ ${ }^{2}$ Department of Physics, University of California, Santa Barbara, California 93106, USA
}

(Received 27 July 2020; accepted 12 February 2021; published 19 March 2021)

\begin{abstract}
In multimode optomechanical systems, the mechanical modes can be coupled via the radiation pressure of the common optical mode, but the fidelity of the state transfer is limited by the optical cavity decay. Here we demonstrate stimulated Raman adiabatic passage (STIRAP) in optomechanics, where the optical mode is not populated during the coherent state transfer between the mechanical modes avoiding this decay channel. We show a state transfer of a coherent mechanical excitation between vibrational modes of a membrane in a high-finesse optical cavity with a transfer efficiency of $86 \%$. Combined with exceptionally high mechanical quality factors, STIRAP between mechanical modes can enable generation, storage, and manipulation of long-lived mechanical quantum states, which is important for quantum information science and for the investigation of macroscopic quantum superpositions.
\end{abstract}

DOI: $10.1103 /$ PhysRevLett.126.113601

Stimulated Raman adiabatic passage (STIRAP) describes adiabatic population transfer between two states coherently coupled via a mediating state that remains unoccupied. This renders STIRAP robust against loss and noise in the mediating state, leading to profound applications in atomic- and molecular-optics research [1,2], trapped-ion physics [3], superconducting circuits [4], other solid-state systems [5,6], optics [7], in entanglement generation [8,9] and qubit operations [10]. STIRAP in optomechanics has been proposed for optical frequency conversion with a mechanical mode being the mediating state, where the fidelity of the state transfer is not deteriorated by the residual thermal noise of the mechanical mode $[11,12]$ and for a mechanical state transfer through the common optical mode [13].

State transfer between nondegenerate mechanical modes was demonstrated in Refs. $[14,15]$ where the beating between two driving light fields bridges the frequency difference of the modes. The motions of the modes modulate the intracavity light fields creating motional sidebands [16]. This transfer scheme relies on the matched motional sidebands and requires the detuning of the driving fields to be much higher than the mechanical frequencies [17]. In this case the other unmatched motional sidebands are of similar amplitudes as the matched ones and cause incoherent driving or cooling of the mechanical modes, limiting the state transfer fidelity. In optomechanical STIRAP in the sideband-resolved regime the loss due to the unmatched motional sidebands can be made negligibly small by choosing the detuning of the two driving light fields equal to the frequencies of the mechanical modes. In this case the two matched sidebands at the cavity resonance interfere destructively driving the state transfer and the other motional sidebands have much smaller amplitudes.
This strongly decreases the unwanted effects of the unmatched motional sidebands and allows the state transfer fidelity to approach unity in the quantum regime.

Figure 1(a) shows the basic $\Lambda$-type arrangement of three levels typical for STIRAP. In the triply rotating frame at frequencies $\omega_{i}=E_{i} / \hbar$ for states $\psi_{i}, i=1,2,3$, the Hamiltonian is
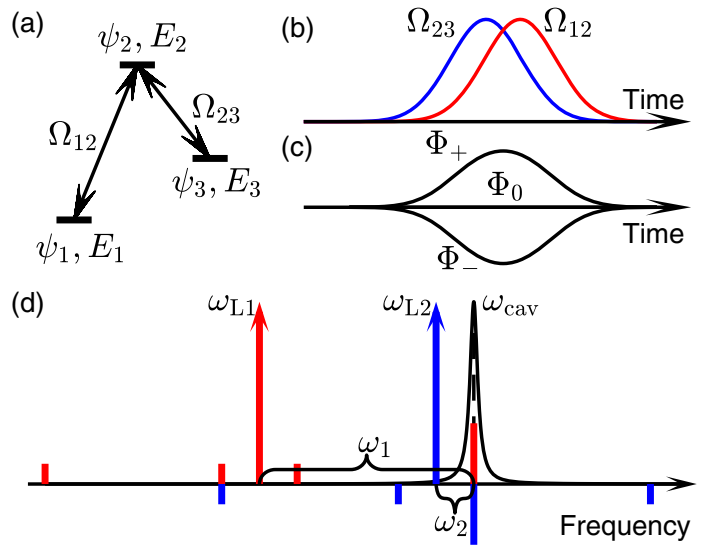

FIG. 1. STIRAP scheme in optomechanics. (a) Energy levels diagram. (b) Coupling strengths of the pulse sequence for the driving fields. (c) The resulting energy eigenvalues for the instantaneous Hamiltonian eigenstates. STIRAP explores the properties of $\Phi_{0}(t)$ given in Eq. (2). (d) The optomechanical implementation contains a cavity mode at frequency $\omega_{\text {cav }}$, two driving fields at $\omega_{L 1}$ and $\omega_{L 2}$ and eight motional sidebands due to the mechanical modes at $\omega_{1}$ and $\omega_{2}$ on the driving fields, red bars corresponding to the sidebands on $\omega_{L 1}$ and blue bars corresponding to the sidebands on $\omega_{L 2}$. Two sidebands match $\omega_{\text {cav }}$. In the case of $\Phi_{0}(t)$ the states $\psi_{1}$ and $\psi_{3}$ are out of phase leading to destructive interference of the sidebands that overlap with $\omega_{\text {cav }}$. 


$$
\hat{H}(t)=\frac{\hbar}{2}\left(\begin{array}{ccc}
0 & \Omega_{12}(t) & 0 \\
\Omega_{12}(t) & 0 & \Omega_{23}(t) \\
0 & \Omega_{23}(t) & 0
\end{array}\right),
$$

with $\Omega_{12}$ and $\Omega_{23}$ the Rabi frequencies resulting from two driving fields at frequencies $\left(E_{2}-E_{1}\right) / \hbar$ and $\left(E_{2}-E_{3}\right) / \hbar$. One of the three instantaneous eigenstates has eigenvalue 0 and does only include states $\psi_{1}$ and $\psi_{3}$ :

$$
\Phi_{0}(t)=\cos \theta(t) \psi_{1}-\sin \theta(t) \psi_{3},
$$

with $\tan \theta(t)=\Omega_{12}(t) / \Omega_{23}(t)$. The existence of this "dark" state in optomechanics has been firstly demonstrated in Ref. [18]. STIRAP is based on the adiabatic following of $\Phi_{0}(t)$ by slowly varying $\theta(t)$ from $\theta(-\infty)=0$ to $\theta(\infty)=\pi / 2$. Thus, the system can be adiabatically transferred from $\psi_{1}$ to $\psi_{3}$ never occupying state $\psi_{2}$. Figure 1(b) shows a driving pulse sequence satisfying this requirement and Fig. 1(c) shows the energy eigenvalues corresponding to the three eigenstates $\Phi_{+}(t), \Phi_{0}(t)$, and $\Phi_{-}(t)$. This driving pulse sequence together with the adiabaticity condition $\sqrt{\Omega_{12}(t)^{2}+\Omega_{23}(t)^{2}} \gg \dot{\theta}$ prevents the lossy mediating state from being occupied throughout the transfer process.

The Hamiltonian in Eq. (1) can be realized in multimode optomechanics $[11,12]$ where states 1 and 3 are mechanical excitations with frequencies $\omega_{1}$ and $\omega_{2}$ and state 2 is an optical cavity mode at $\omega_{\text {cav }}$, see Fig. 1(d). Two optical driving fields at $\omega_{L i}=\omega_{\text {cav }}-\omega_{i}$ for $i=1,2$ are introduced in order to create the beam splitter interaction $\hat{a} \hat{b}_{i}^{\dagger}+\hat{a}^{\dagger} \hat{b}_{i}$ that couples the mechanical modes to the cavity mode, where $\hat{a}\left(\hat{a}^{\dagger}\right)$ and $\hat{b}_{i}\left(\hat{b}_{i}^{\dagger}\right)$ are the photon and phonon annihilation (creation) operators. The optical mode can be represented by the operator $\hat{a}=\bar{\alpha}+\delta \hat{a}$, where $\bar{\alpha}$ is the average coherent amplitude due to the driving optical fields and $\delta \hat{a}$ is the fluctuating term [16]. Each mechanical mode produces two sidebands on each optical field. Because of resonance with the cavity, the two sidebands with frequencies $\omega_{\text {cav }}$ acquire much larger amplitudes than the other sidebands. Taking into account only those two sidebands and including mechanical and optical loss rates, $\Gamma_{i}$ and $\kappa$, the time evolution of the state vector $\psi(t)=$ $\left[\hat{b}_{1}(t), \delta \hat{a}(t), \hat{b}_{2}(t)\right]^{T}$ is given by

$$
i \frac{d \psi(t)}{d t}=\left(\begin{array}{ccc}
-i \frac{\Gamma_{1}}{2} & g_{1}(t) & 0 \\
g_{1}(t) & -i \frac{\kappa}{2} & g_{2}(t) \\
0 & g_{2}(t) & -i \frac{\Gamma_{2}}{2}
\end{array}\right) \psi(t) .
$$

Here, the rotating wave approximation has been used and it is valid in the linearized regime of cavity optomechanics [16]. $g_{i}(t)$ is the optomechanical multiphoton coupling for mechanical modes $i=1,2, g_{i}=g_{i 0} \bar{\alpha}_{i}$, where $g_{i 0}$ is single photon coupling and $\bar{\alpha}_{i}$ is the driving field at $\omega_{L i}$, see
Supplemental Material [19]. Equation (3) is valid in the sideband resolved regime together with the requirement $\left|\omega_{1}-\omega_{2}\right| \gg \kappa$ and is identical to Eq. (1) in the absence of losses and with the Rabi frequencies $\Omega_{12}$ and $\Omega_{23}$ corresponding to $2 g_{1}$ and $2 g_{2}$.

Experimentally we demonstrate the state transfer in the membrane-in-the-middle (MIM) configuration [32], where a membrane with low optical absorption is placed in the center of a high-finesse optical cavity with $\kappa / 2 \pi=54 \mathrm{kHz}$ (including membrane), see Fig. 2. A displacement of the membrane along the optical axis leads to a shift in the optical cavity transmission described by the interaction Hamiltonian $\hat{H}_{\text {int }}=-\hbar g_{0} \hat{a}^{\dagger} \hat{a}\left(\hat{b}+\hat{b}^{\dagger}\right)$, where $g_{0}$ is the single photon optomechanical coupling [16]. The membrane is a highly stressed $25 \mathrm{~nm}$ thick SiN film lithographically patterned with a phononic crystal with a defect in its center suspended on a silicon frame [33]. There are two types of mechanical modes: whole membrane drumhead modes and modes localized near the phononic crystal defect with frequencies in the phononic crystal band gap. Vibrational energy of the drumhead modes is mainly lost in the bending regions where the membrane is connected to the frame $[33,34]$. The modes localized near the defect possess enhanced quality factors by 1-2 orders of magnitude compared to the drumhead modes [33]. We demonstrate STIRAP between the fundamental mode of the defect with frequency $\omega_{1} / 2 \pi=1.25 \mathrm{MHz}$ and quality factor $Q=$ $1.3 \times 10^{7}$ [mode 1, Fig. 2(b)], and the 3,3 drumhead mode with $\omega_{2} / 2 \pi=0.22 \mathrm{MHz}$ and $Q=1.2 \times 10^{6}$ [mode 2, Fig. 2(c)]. The modes are coupled to the optical cavity with single-photon couplings of $g_{01} / 2 \pi=1.5 \pm 0.1 \mathrm{~Hz}$ and $g_{02} / 2 \pi=1.0 \pm 0.1 \mathrm{~Hz}$, respectively. In addition to these modes possessing relatively large single photon

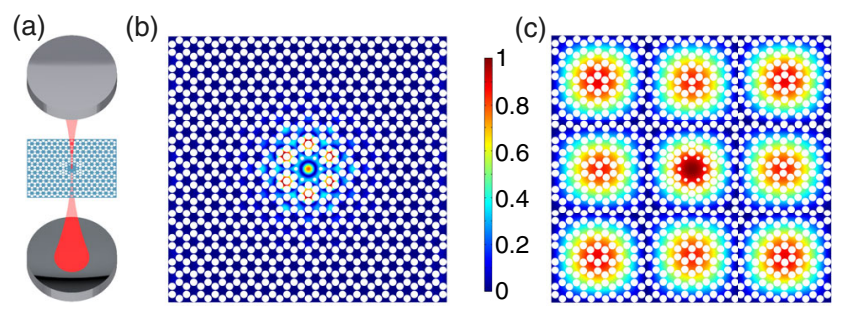

FIG. 2. Optomechanical setup. (a) A transparent dielectric membrane patterned with a phononic crystal is placed in the middle of a high-finesse optical cavity. Shift of the membrane along the axis of the cavity changes the cavity resonance frequency, causing coupling of light in the cavity to vibrational modes of the membrane. (b) Simulated displacement of a mechanical mode of the defect of the phononic crystal. The mode is localized as its frequency lies in the band gap (mode 1, initially excited). (c) Simulated displacement of the 3,3 drumhead mode of the full membrane (mode 2). This second mode was selected because it has an appropriate mechanical frequency and quality factor and has a maximum amplitude at the center. This allows both modes to be aligned for optimal coupling to the same cavity mode. 
coupling, quality factors, and frequency separation, there are no other mechanical modes in the range of $\sim 1 / \sigma$ from $\omega_{1}$ and $\omega_{2}$, where $\sigma$ defines the width of the driving pulses. The latter requirement guaranties that modes 1 and 2 are not coupled to other modes during the transfer. STIRAP is very sensitive to the double-photon detuning $\Delta_{2 \mathrm{ph}}=$ $\left(\omega_{L 1}+\omega_{1}\right)-\left(\omega_{L 2}+\omega_{2}\right)$ [35], therefore the two optical fields are created by amplitude modulation of light from a single $1064 \mathrm{~nm}$ laser using an acousto-optic modulator $(\mathrm{AOM})$. As a result the laser phase noise does not limit the transfer efficiency [36]. Because of the nonlinear response of the AOM the detuning of this single laser light tone is chosen such that harmonics of the ac voltage sent to the AOM have a negligible effect on the transfer efficiency (see Supplemental Material). The membrane is in a vacuum chamber with pressure below $10^{-6} \mathrm{mbar}$ at room temperature.

STIRAP with parameters tuned for maximum phonon number state transfer efficiency is shown in Fig. 3. The measurement of a typical transfer process has the following sequence: mode 1 is excited to an amplitude much higher than its thermal occupation by applying an ac voltage in resonance with the mechanical frequency to a needle positioned close to the center of the membrane. During its free decay the two optical pulses are sent which transfers the excitation of mode 1 to mode 2 . The transfer starts with the beginning of pulse 1 (red) and finishes with the end of pulse 2 (blue), these moments are denoted by dashed vertical lines. The transfer efficiency is calculated as the ratio of the number of phonons in mechanical mode 2 at the end of the transfer to the number of phonons in mechanical mode 1 at the beginning of the transfer (black stars). A theoretical model without free fit parameters was developed in the classical limit to simulate the transfer process taking into account the corrections due to the other sidebands and the measured profiles of the light pulses (see Supplemental Material), and shows excellent agreement to the experimental data in Fig. 3(a). Simulations show that the average rate of loss through the optical mode is $\sim 1 \mathrm{~Hz}$ in the dark state during the transfer. We observe small variations in the frequencies of the mechanical modes with each STIRAP sequence. To account for these variations, we measure the mechanical frequencies in thermal motion and adjust the values of the mechanical frequencies for the driving pulse generation accordingly before each STIRAP sequence.

In our realization of STIRAP using coherent state populations, i.e., in the classical regime, the phases of the mechanical modes during the transfer can be continuously monitored, see Fig. 3(b). There are four time domains with distinct behavior of phases: in domain 1 $g_{1}(t)=0$ and the phase of mode 1 is defined by the excitation used to drive it, while mode 2 is in its thermal motion, thus the difference between the phases is random; in domain 2 STIRAP starts and the phase of mode 2 adjusts itself until the sidebands at $\omega_{\text {cav }}$ become $\pi$ out of phase; in
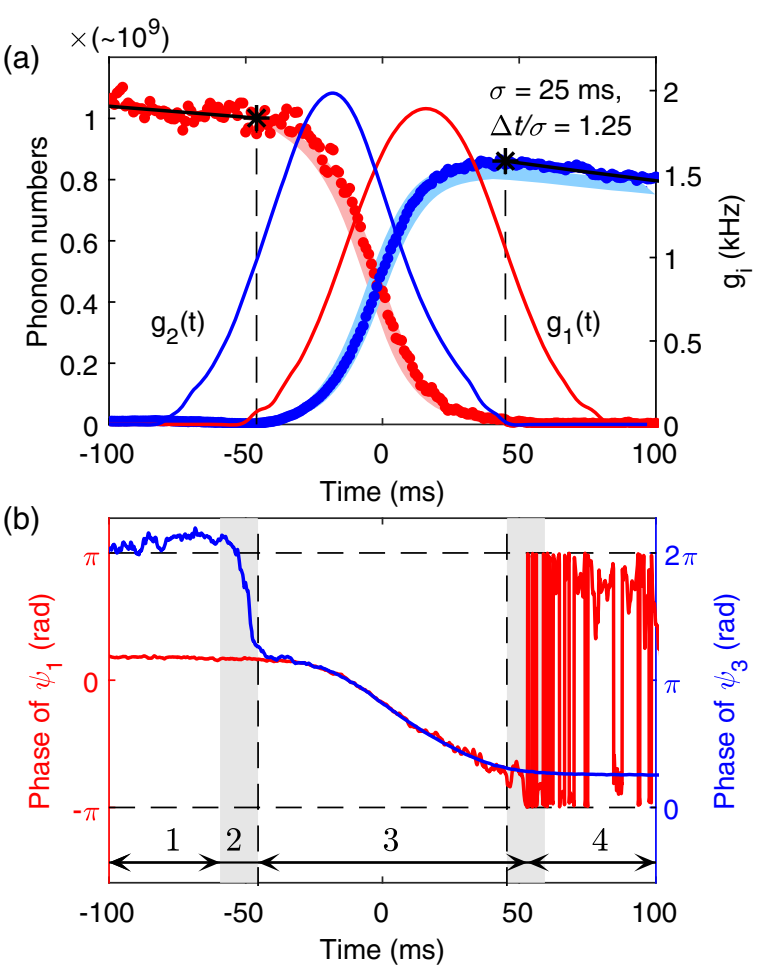

FIG. 3. Experimental optomechanical STIRAP. (a) Left scale: phonon number as a function of time, red dots correspond to averaged measurements for mode $1\left(\psi_{1}\right)$, blue dots for mode 2 $\left(\psi_{3}\right)$. The prefactor $10^{9}$ is a rough estimate. Light red and light blue regions represent the phonon populations with statistical uncertainties (1 standard deviation) obtained from simulations without free fit parameters. Right scale: multiphoton optomechanical coupling strengths, calculated from measured pulse intensities. The driving field pulses have a nearly Gaussian profile with the standard deviation parameter $\sigma$ and separation $\Delta t$, but their beginning and ending are smoothly truncated to zero. Black stars correspond to the phonon populations used to calculate transfer efficiency ( $5 \%$ of the peak voltage sent to the AOM). (b) Measured phases of mode 1 (red) and mode 2 (blue) in the rotating frame.

domain 3 the phase of the locked mechanical modes changes due to the optomechanically induced frequency shift from field $\omega_{L 2}$ (unmatched sidebands); in domain 4 the read-out signal of mode 1 becomes much less than the read-out noise.

Next we investigate the dependence of the transfer efficiency on the parameters of the process. First the time delay between the optical pulses $\Delta t$ is varied, see Fig. 4 . The adiabaticity condition becomes more and more violated when the separation between the pulses is too small or too large, leading to decreasing efficiency. Then the duration of the pulses $\sigma$ is varied while keeping the time delay $\Delta t$ optimal. The adiabaticity condition is satisfied increasingly better with longer pulses such that for pulses with $\sigma=100 \mathrm{~ms}$ only $2 \%$ of the initial phonon population in mode 1 is lost through the population and decay of the optical mode. Nevertheless the efficiency starts to decrease 

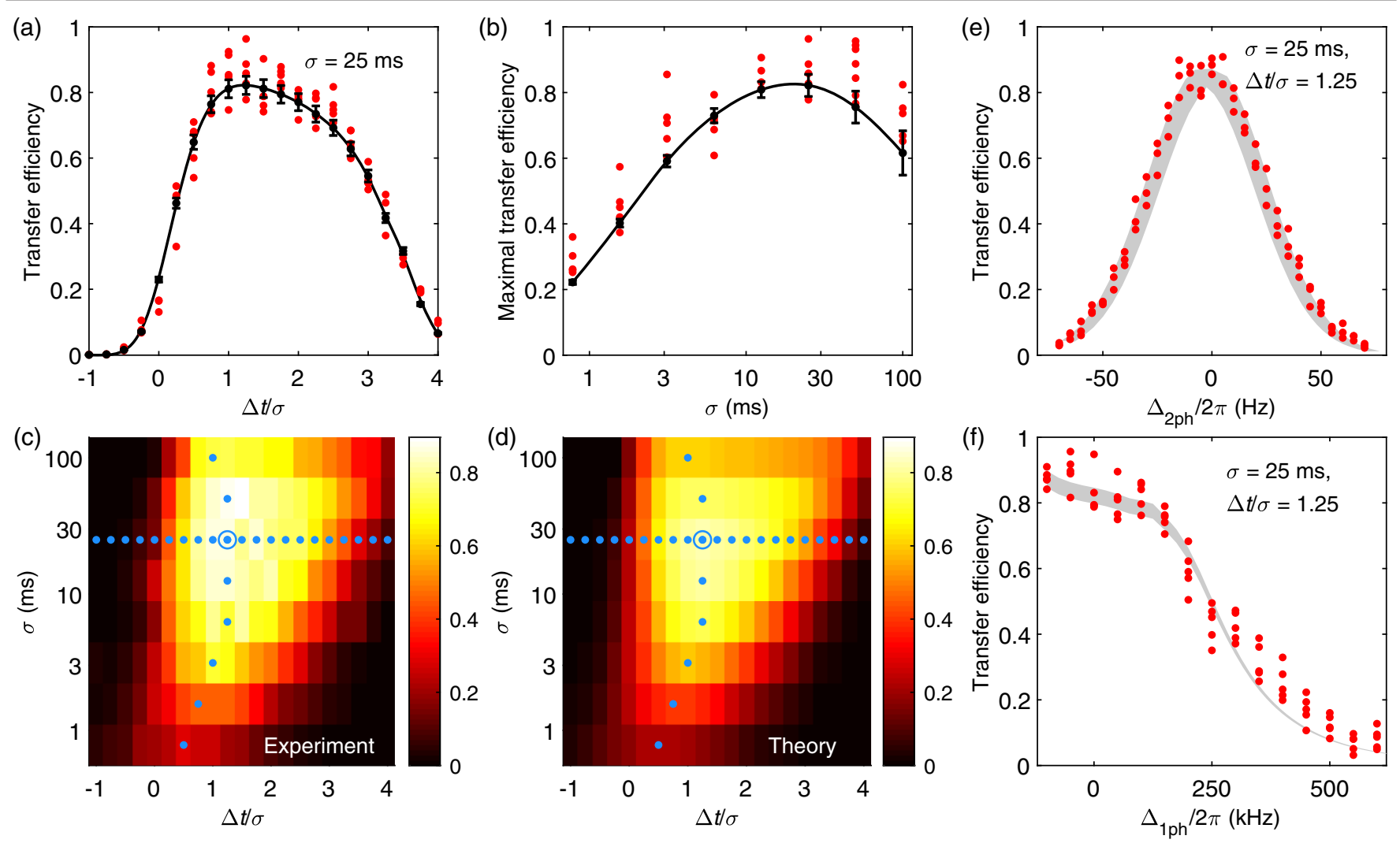

FIG. 4. Transfer efficiencies under different parameters of the optical pulses. (a) The transfer efficiency as a function of the ratio of the delay between the pulses $\Delta t$ and the Gaussian pulse width $\sigma$. Positive values of $\Delta t$ correspond to the case that the field at $\omega_{L 2}$ is applied before the field at $\omega_{L 1}$. (b) Maximal transfer efficiencies as a function of $\sigma$. In (a) and (b) the red dots show measured efficiencies in individual runs, black dots are the simulated efficiencies, and the black lines are guides to the eye. The increasing error bars for larger $\sigma$ in (b) are due to observed but not accounted for small nonlinear and heating effects, see Supplemental Material. (c) and (d) show the experimental (c) and predicted (d) transfer efficiency as a function of the Gaussian parameter $\sigma$ and separation $\Delta t$. The horizontal row of dots in (c) and (d) correspond to the data shown in (a), while the vertical row of dots correspond to the data shown in (b). The transfer process for the parameters corresponding to the open circle in (c) and (d) is shown in Fig. 3(e). (e) The efficiency as a function of the twophoton detuning $\Delta_{2 \mathrm{ph}}$ with zero single-photon detuning. (f) The efficiency as a function of the single-photon detuning $\Delta_{1 \mathrm{ph}}$ with zero two-photon detuning. In (e) and (f) the red circles are measured efficiencies in individual runs, and the shaded regions are simulated efficiencies with statistical uncertainties. The simulated curve in (e) has a frequency correction of $4 \mathrm{~Hz}$ caused by small nonlinear and heating effects, see Supplemental Material.

for $\sigma \gtrsim 25 \mathrm{~ms}$ due to the mechanical decay of the modes, setting the upper bound on the transfer efficiency. The solid curves in Figs. 4(a) and 4(b) are numerical results and Figs. 4(c) and 4(d) compare experiment and simulations for varying $\Delta t$ and $\sigma$. We observe an increasing discrepancy between measured and simulated data for the state transfer with $\sigma \gtrsim 25 \mathrm{~ms}$. This is caused by membrane heating from the driving pulses and by the defect mode frequency dependence on the amplitude of the full membrane 3,3 mode, see Supplemental Material.

A signature of STIRAP [35] is strong sensitivity of the transfer efficiency to the two-photon detuning $\Delta_{2 \text { ph }}=$ $\left(\omega_{L 1}+\omega_{1}\right)-\left(\omega_{L 2}+\omega_{2}\right)$ given $\Delta_{1 \mathrm{ph}}=0$, compared to the sensitivity to the single-photon detuning $\Delta_{1 \mathrm{ph}}=\omega_{\mathrm{cav}}-$ $\left(\omega_{L 1}+\omega_{1}\right)$ given $\Delta_{2 \mathrm{ph}}=0$, Figs. 4(e) and 4(f). The frequency scale for the two-photon detuning is set by the duration of the transfer process: $\Delta_{2 \mathrm{ph}} \sim \pi / T_{\text {transfer }}$, implying that the sidebands at $\omega_{\text {cav }}$ accumulate a phase difference of $\pi$ during the transfer and consequently no longer interfere destructively. The frequency scale for $\Delta_{1 \mathrm{ph}}$ is set by the optical cavity linewidth $\kappa$ : nonzero $\Delta_{1 \mathrm{ph}}$ leads to changes in the intracavity light fields intensities and in the amplitudes of the sidebands.

The highest phonon number transfer efficiency we observe in our system is $86 \% \pm 3 \%$. The highest demonstrated state transfer efficiencies in other systems are: transmon qubit 83\% [4]; Bose-Einstein condensate of atoms $87 \%$ [37]; trapped ions $90 \%$ [3]; superconducting Xmon qutrit 96\% [38]; doped crystals 98\% $\pm 2 \%$ [39]; atom beams $98 \% \pm 2 \%$ [40]. In general, the STIRAP scheme in optomechanics can result in transfer efficiencies close to unity provided that the difference between the frequencies of the mechanical modes is much larger than the cavity linewidth, while being in the weak coupling 
regime, with peak multiphoton optomechanical couplings being much larger than the inverse of the transfer duration, and with slow enough mechanical decay. This allows us to formulate the following requirements for optomechanical STIRAP:

$$
\left|\omega_{1}-\omega_{2}\right| \gg \kappa \gg \max g_{i}(t) \gg 2 \pi / T_{\text {transfer }} \gg \Gamma_{i} .
$$

This set of stringent requirements applies both to the classical and the quantum regime of STIRAP in optomechanics. Other experimental challenges are the accurate control of one- and two-photon detunings, circumventing detrimental effects of the unmatched sidebands, and proving stable subwavelength positioning of the membrane to maximize the coupling strength.

Further we show that state transfer via STIRAP of a single-phonon Fock state can in principle be observed experimentally with the same membranes in a cryogenic setting, for details see Supplemental Material [19]. We provide a full quantum treatment of the protocol for such a state transfer including known sources of noise and unwanted effects: thermalization to the environment, heating by the laser light fields, presence of other nearby membrane modes, realistic overall detection efficiency, and dark count rate of a single photon detector. We consider STIRAP between two modes of the defect of the phononic crystal in the membrane, with quality factors of $10^{9}$ [41], resulting in a thermal decoherence time [16] of approximately $5 \mathrm{~ms}$ at $1 \mathrm{~K}$. In laser cooling experiments $[42,43]$ the steady state temperature of similar membranes was observed to be less than $0.5 \mathrm{~K}$ above the cryostat base temperature when being sideband cooled, thus we adopt $1 \mathrm{~K}$ as a conservative estimate for the membrane temperature due to laser heating.

The protocol consists of the following steps: both modes are sideband cooled to an average phonon occupation $\bar{n}=0.1$; detection of a Stokes photon from a short bluedetuned pulse projects the state of mode 1 to a state close to a single-phonon Fock state; the STIRAP pulse sequence is sent; the state of the modes is read out by a short reddetuned pulse through detection of anti-Stokes photons. It is essential to filter out the strong pump light fields and to send the scattered photons to a single photon detector with high enough overall detection efficiency. Based on demonstrated experimental parameters we calculate that a single-phonon Fock state can be transferred with fidelity of $60 \%$.

In conclusion, in this Letter we have shown the first optomechanical implementation of STIRAP and demonstrated a maximum phonon number state transfer efficiency of $86 \% \pm 3 \%$. The efficiency is benchmarked against variation in the STIRAP pulse duration and separation as well as against the STIRAP single- and two-photon detuning and is found to be in good agreement with theory. Our quantum simulations show that STIRAP of a single phonon Fock state is feasible to observe with the demonstrated technology. Furthermore, modified versions of STIRAP (fractional STIRAP [44], tripod STIRAP [45]) can be used to create and detect entangled mechanical states. Therefore, STIRAP in optomehanics can play an important role in quantum information protocols and in generating macroscopic superposition states.

We would like to thank K. Heeck, H. van der Meer, and S. Sonar for support of this project. This work was supported by the UC Santa Barbara NSF Quantum Foundry funded via the Q-AMASE-i program Grant No. DMR1906325, and the NWO Gravitation-grant Quantum Software Consortium-024.003.037, and the NWO Vrij Programma Grant No. QUAKE—680.92.18.04.

*vfedoseev@physics.leidenuniv.nl

[1] U. Gaubatz, P. Rudecki, M. Becker, S. Schiemann, M. Külz, and K. Bergmann, Population switching between vibrational levels in molecular beams, Chem. Phys. Lett. 149, 463 (1988).

[2] P. Pillet, C. Valentin, R.-L. Yuan, and J. Yu, Adiabatic population transfer in a multilevel system, Phys. Rev. A 48, 845 (1993).

[3] J. L. Sørensen, D. Møller, T. Iversen, J. B. Thomsen, F. Jensen, P. Staanum, D. Voigt, and M. Drewsen, Efficient coherent internal state transfer in trapped ions using stimulated Raman adiabatic passage, New J. Phys. 8, 261 (2006).

[4] K. S. Kumar, A. Vepsälaïnen, S. Danilin, and G. S. Paraoanu, Stimulated Raman adiabatic passage in a three-level superconducting circuit, Nat. Commun. 7, 10628 (2016).

[5] H. Goto and K. Ichimura, Observation of coherent population transfer in a four-level tripod system with a rare-earth-metalion-doped crystal, Phys. Rev. A 75, 033404 (2007).

[6] D. A. Golter and H. Wang, Optically Driven Rabi Oscillations and Adiabatic Passage of Single Electron Spins in Diamond, Phys. Rev. Lett. 112, 116403 (2014).

[7] S. Longhi, Quantum-optical analogies using photonic structures, Laser Photonics Rev. 3, 243 (2009).

[8] J. Simon, H. Tanji, S. Ghosh, and V. Vuletic, Single-photon bus connecting spin-wave quantum memories, Nat. Phys. 3, 765 (2007).

[9] H.-S. Chang, Y. P. Zhong, A. Bienfait, M.-H. Chou, C. R. Conner, É. Dumur, J. Grebel, G. A. Peairs, R. G. Povey, K. J. Satzinger, and A. N. Cleland, Remote Entanglement Via Adiabatic Passage Using a Tunably-Dissipative Quantum Communication System, Phys. Rev. Lett. 124, 240502 (2020).

[10] K. Toyoda, K. Uchida, A. Noguchi, S. Haze, and S. Urabe, Realization of holonomic single-qubit operations, Phys. Rev. A 87, 052307 (2013).

[11] Y. D. Wang and A. A. Clerk, Using Interference for High Fidelity Quantum State Transfer in Optomechanics, Phys. Rev. Lett. 108, 153603 (2012).

[12] L. Tian, Adiabatic State Conversion and Pulse Transmission in Optomechanical Systems, Phys. Rev. Lett. 108, 153604 (2012). 
[13] D. Garg, A. K. Chauhan, and A. Biswas, Adiabatic transfer of energy fluctuations between membranes inside an optical cavity, Phys. Rev. A 96, 023837 (2017).

[14] M. J. Weaver, F. Buters, F. Luna, H. Eerkens, K. Heeck, S. De Man, and D. Bouwmeester, Coherent optomechanical state transfer between disparate mechanical resonators, Nat. Commun. 8, 824 (2017).

[15] H. Xu, L. Jiang, A. A. Clerk, and J. G. Harris, Nonreciprocal control and cooling of phonon modes in an optomechanical system, Nature (London) 568, 65 (2019).

[16] M. Aspelmeyer, T. J. Kippenberg, and F. Marquardt, Cavity optomechanics, Rev. Mod. Phys. 86, 1391 (2014).

[17] L. F. Buchmann and D. M. Stamper-Kurn, Nondegenerate multimode optomechanics, Phys. Rev. A 92, 013851 (2015).

[18] C. Dong, V. Fiore, M. C. Kuzyk, and H. Wang, Optomechanical dark mode, Science 338, 1609 (2012).

[19] See Supplemental Material at http://link.aps.org/ supplemental/10.1103/PhysRevLett.126.113601 for a detailed description of measurements and the complete theoretical model, which includes Refs. [20-31].

[20] N. V. Vitanov, K.-A. Suominen, and B. W. Shore, Creation of coherent atomic superpositions by fractional stimulated raman adiabatic passage, J. Phys. B 32, 4535 (1999).

[21] M. J. Weaver, D. Newsom, F. Luna, W. Löffler, and D. Bouwmeester, Phonon interferometry for measuring quantum decoherence, Phys. Rev. A 97, 063832 (2018).

[22] I. Galinskiy, Y. Tsaturyan, M. Parniak, and E. S. Polzik, Phonon counting thermometry of an ultracoherent membrane resonator near its motional ground state, Optica 7, 718 (2020).

[23] J. Johansson, P. Nation, and F. Nori, QuTiP: An open-source PYTHON framework for the dynamics of open quantum systems, Comput. Phys. Commun. 183, 1760 (2012).

[24] H. Flayac and V. Savona, Heralded Preparation and Readout of Entangled Phonons in a Photonic Crystal Cavity, Phys. Rev. Lett. 113, 143603 (2014).

[25] Y. Zhai, Z. X. Chen, and Q. Lin, Efficient ground state cooling of a mechanical resonator in a membrane-in-the-middle system by a single drive, J. Opt. Soc. Am. B 37, 956 (2020).

[26] H. Shibata, K. Shimizu, H. Takesue, and Y. Tokura, Ultimate low system dark-count rate for superconducting nanowire single-photon detector, Opt. Lett. 40, 3428 (2015).

[27] H. Shibata, K. Shimizu, H. Takesue, and Y. Tokura, Superconducting nanowire single-photon detector with ultralow dark count rate using cold optical filters, Appl. Phys. Express 6, 072801 (2013).

[28] C. Fleming, N. I. Cummings, C. Anastopoulos, and B. L. Hu, The rotating-wave approximation: consistency and applicability from an open quantum system analysis, J. Phys. A 43, 405304 (2010).

[29] R. W. Drever, J. L. Hall, F. V. Kowalski, J. Hough, G. M. Ford, A. J. Munley, and H. Ward, Laser phase and frequency stabilization using an optical resonator, Appl. Phys. B 31, 97 (1983).

[30] R. Riedinger, A. Wallucks, I. Marinković, C. Löschnauer, M. Aspelmeyer, S. Hong, and S. Gröblacher, Remote quantum entanglement between two micromechanical oscillators, Nature (London) 556, 473 (2018).

[31] A. M. Jayich, J. C. Sankey, B. M. Zwickl, C. Yang, J. D. Thompson, S. M. Girvin, A. A. Clerk, F. Marquardt, and J. G. Harris, Dispersive optomechanics: A membrane inside a cavity, New J. Phys. 10, 095008 (2008).

[32] J. D. Thompson, B. M. Zwickl, A. M. Jayich, F. Marquardt, S. M. Girvin, and J. G. Harris, Strong dispersive coupling of a high-finesse cavity to a micromechanical membrane, Nature (London) 452, 72 (2008).

[33] Y. Tsaturyan, A. Barg, E. S. Polzik, and A. Schliesser, Ultracoherent nanomechanical resonators via soft clamping and dissipation dilution, Nat. Nanotechnol. 12, 776 (2017).

[34] S. Schmid, K. D. Jensen, K. H. Nielsen, and A. Boisen, Damping mechanisms in high-Q micro and nanomechanical string resonators, Phys. Rev. B 84, 165307 (2011).

[35] N. V. Vitanov, A. A. Rangelov, B. W. Shore, and K. Bergmann, Stimulated Raman adiabatic passage in physics, chemistry, and beyond, Rev. Mod. Phys. 89, 015006 (2017).

[36] A. Kuhn, G. W. Coulston, G. Z. He, S. Schiemann, K. Bergmann, and W. S. Warren, Population transfer by stimulated Raman scattering with delayed pulses using spectrally broad light, J. Chem. Phys. 96, 4215 (1992).

[37] M. Dupont-Nivet, M. Casiulis, T. Laudat, C. I. Westbrook, and S. Schwartz, Microwave-stimulated Raman adiabatic passage in a Bose-Einstein condensate on an atom chip, Phys. Rev. A 91, 053420 (2015).

[38] H. K. Xu, C. Song, W. Y. Liu, G. M. Xue, F. F. Su, H. Deng, Y. Tian, D. N. Zheng, S. Han, Y. P. Zhong, H. Wang, Y. X. Liu, and S. P. Zhao, Coherent population transfer between uncoupled or weakly coupled states in ladder-type superconducting qutrits, Nat. Commun. 7, 11018 (2016).

[39] J. Klein, F. Beil, and T. Halfmann, Experimental investigations of stimulated Raman adiabatic passage in a doped solid, Phys. Rev. A 78, 033416 (2008).

[40] H. Theuer and K. Bergmann, Atomic beam deflection by coherent momentum transfer and the dependence on weak magnetic fields, Eur. Phys. J. D 2, 279 (1998).

[41] M. Rossi, D. Mason, J. Chen, Y. Tsaturyan, and A. Schliesser, Measurement-based quantum control of mechanical motion, Nature (London) 563, 53 (2018).

[42] R. W. Peterson, T. P. Purdy, N. S. Kampel, R. W. Andrews, P. L. Yu, K. W. Lehnert, and C. A. Regal, Laser Cooling of a Micromechanical Membrane to the Quantum Backaction Limit, Phys. Rev. Lett. 116, 063601 (2016).

[43] M. Underwood, D. Mason, D. Lee, H. Xu, L. Jiang, A. B. Shkarin, K. Børkje, S. M. Girvin, and J. G. Harris, Measurement of the motional sidebands of a nanogram-scale oscillator in the quantum regime, Phys. Rev. A 92, 061801 (2015).

[44] P. Marte, P. Zoller, and J. L. Hall, Coherent atomic mirrors and beam splitters by adiabatic passage in multilevel systems, Phys. Rev. A 44, R4118 (1991).

[45] R. Unanyan, M. Fleischhauer, B. W. Shore, and K. Bergmann, Robust creation and phase-sensitive probing of superposition states via stimulated Raman adiabatic passage (STIRAP) with degenerate dark states, Opt. Commun. 155, 144 (1998). 